\title{
Successful Microsurgical Reconstruction for Limb Salvage in a COVID-19-Positive Patient: A Case Report
}

Christopher M. Fleury, MD ${ }^{1} \quad$ Brian L. Chang, MD $\quad$ Robert P. Slamin, MD ${ }^{1} \quad$ Jonathan A. Schwitzer, MD ${ }^{1}$ Arjun Kanuri, MD ${ }^{1}$ Derek L. Masden, MD²

\author{
${ }^{1}$ Department of Plastic Surgery, MedStar Georgetown University \\ Hospital, Washington, District of Columbia \\ ${ }^{2}$ Department of Plastic Surgery, MedStar Washington Hospital \\ Center, Washington, District of Columbia
}

\begin{abstract}
Address for correspondence Christopher M. Fleury, MD, Department of Plastic Surgery, MedStar Georgetown University Hospital, 3800 Reservoir Rd NW, Washington, DC 20007 (e-mail: christopher.michael.fleury@gmail.com).
\end{abstract}

J Reconstr Microsurg Open 2021;6:e40-e44.

\begin{abstract}
Keywords

- microsurgery

- COVID-19

- limb salvage

Background Although the novel 2019 coronavirus (COVID-19) pandemic delayed elective microsurgical reconstruction cases, traumatic injuries requiring microsurgical reconstruction for limb salvage still presented to our hospital. Recent data suggests a link between COVID-19 and increased venous and arterial thromboembolic events, theoretically increasing the risk of microvascular anastomotic thrombosis and flap failure.

Methods We provide a single case report of microsurgical limb salvage in a COVID-19positive patient with a Gustilo IIIb open tibial-fibular fracture.

Results A free latissimus dorsi muscle flap was used to successfully cover a Gustilo IIIb open tibial-fibular fracture with exposed orthopedic hardware and large soft tissue defect, with 5-month follow-up demonstrating a well-healed flap allowing for preserved ambulation.

Conclusion Although there is a theoretical increased incidence of venous and arterial thrombotic microvascular failure in COVID-19-positive patients, microsurgical reconstruction is occasionally the lone option for salvage. Microsurgical reconstruction in this population must be carefully considered, meticulously executed, and closely monitored.
\end{abstract}

The novel 2019 coronavirus disease (COVID-19) pandemic has altered practice patterns and resource utilization across all medical fields, including plastic surgery. In April 2020, there was a mandate by professional societies and hospital systems to delay elective reconstructive microsurgical cases. However, as Janis and co-workers discussed in their June 2020 summary of the practice recommendations for plastic surgeons, guidance on the definition of elective surgery was somewhat vague for plastic surgeons, and trauma reconstruction was considered nonelective if postponement threatened life or limb. ${ }^{1}$ Indeed, traumatic injuries requiring microsurgical reconstruction for limb salvage still present to our hospital systems, and these patients may be COVID-19-positive, with or without symptoms. To further complicate matters, recently published data suggest a link between COVID-19 and a hypercoagulable state, with received

January 7, 2021

accepted after revision

March 3, 2021
DOI https://doi.org/ $10.1055 / \mathrm{s}-0041-1729955$. ISSN 2377-0813.

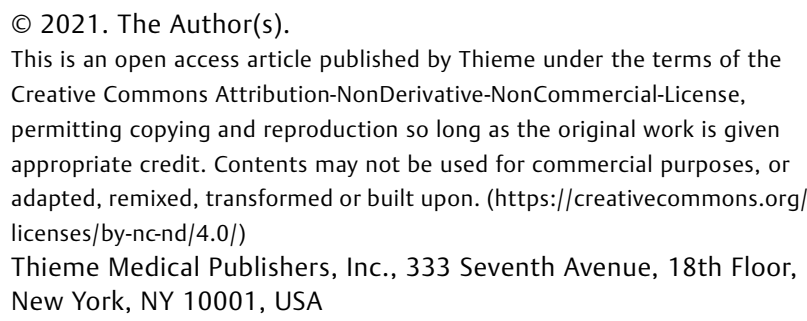


disproportionately high incidence of both venous (deep vein thrombosis [DVT] and pulmonary embolism [PE]) and arterial thrombosis in COVID-19-positive patients, as high as $31 \%$ of 184 patients in intensive care unit in a recent publication, as well as abnormal laboratory coagulation study values in both symptomatic and asymptomatic patients. ${ }^{2-11}$ This hypercoagulable state theoretically increases the thrombosis and failure rate in microsurgical reconstruction, and indeed there have been published reports of free-flap failures in patients who were retrospectively found to be COVID-19positive. $^{12}$ To our knowledge, no report of a successful free flap in a preoperatively known COVID-19-positive patient has been published to date. We present a case of successful microsurgical reconstruction for limb salvage in a Gustilo IIIb open tibial-fibular fracture with exposed orthopaedic hardware in a COVID-19-positive patient, which we performed in May 2020, prior to the widespread knowledge of increased morbidity and mortality associated with repeated intubations in this patient population. ${ }^{13}$ It serves not as a recommendation for microsurgical reconstruction in patients during the period of COVID-positivity but instead as a demonstration of feasibility of such reconstruction, as well as suggestion of a possible postoperative antithrombotic protocol, which proved successful in our case.

\section{Case}

On May 20, 2020, a 44-year-old previously healthy male construction worker presented to our trauma bay after falling 45 feet and landing on his legs. He was hemodynamically stable without any abnormal findings on primary survey. Subsequent orthopaedic workup showed multiple fractures involving the sacrum and pubic symphysis, closed right foot calcaneal fracture and Lisfranc fracture-dislocation, open left calcaneal fracture, and right leg Gustilo IIIb open tibial-fibular shaft fracture with extension into the tibiotalar joint in a pilon pattern and syndesmotic injury (-Fig. 1a,b). According to institutional policy, he was tested for COVID-19 using nasopharyngeal swab PCR (polymerase chain reaction) and found to be positive. He was otherwise asymptomatic. This positive result was confirmed three additional times over the next 2 weeks.

Over the next 13 days, the patient was taken to the operating room three times with orthopaedic surgery for fixation of pelvic fractures, external fixation of lower extremity fractures, and eventual internal fixation of his right tibial and fibular fractures with medial and lateral tibial plates and a flexible intramedullary fibular nail, as well as plating of his right midfoot injuries (-Fig. 1c). The plastic surgery service was consulted intraoperatively during the fixation, given the large soft-tissue defect overlying right leg hardware. The patient was temporized with negative pressure wound therapy. He subsequently underwent diagnostic angiography for preoperative planning, given the high-energy mechanism of injury, which demonstrated patent superficial femoral and popliteal arteries, early occlusion of the anterior tibial artery, patent peroneal artery to the ankle, and patent posterior tibial artery to the foot, effectively demonstrating one vessel runoff to the foot ( $\mathbf{F i g . 2 a , b}$ ). On hospital day 13 , the patient was taken to the operating room by the plastic surgery service and underwent a free left latissimus dorsi muscle flap to the right lower extremity. The artery was handsewn end-to-side into the posterior tibial artery and two venous anastomoses were performed with 2.5 and $2.0 \mathrm{~mm}$ couplers between the donor and recipient venae comitantes. The muscle was covered with overlying splitthickness skin graft harvested from the left thigh ( - Figs. 3ad). The patient received aspirin $325 \mathrm{mg}$ orally preoperatively, and an intraoperative dose of 3,000 units of intravenous heparin was administered prior to pedicle ligation and anastomosis. Postoperatively, he was started on daily aspirin $325 \mathrm{mg}$ orally (in addition to the preoperative dose), and a
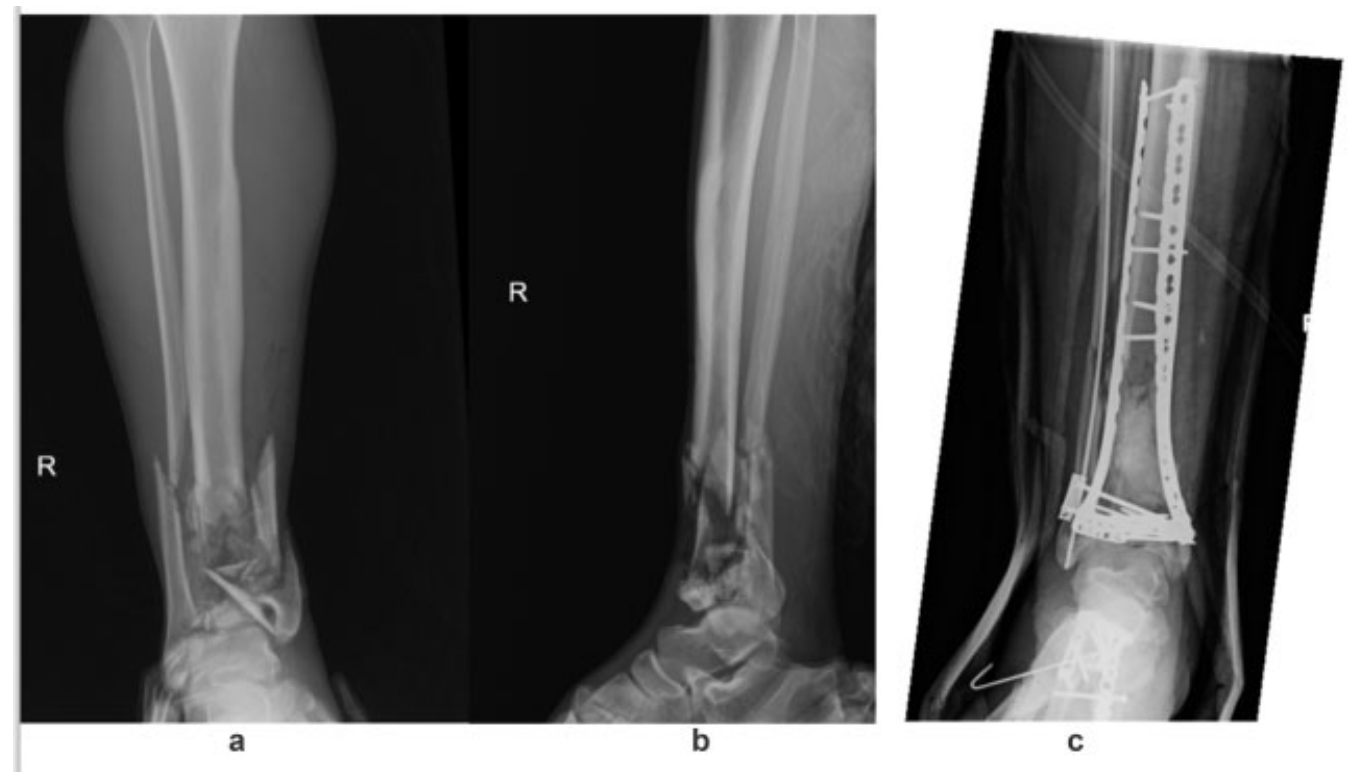

Fig. 1 Radiographs: (a) anteroposterior view of initial right leg injury; (b) lateral view of initial right leg injury; and (c) hardware after definitive internal fixation of orthopaedic injury. 


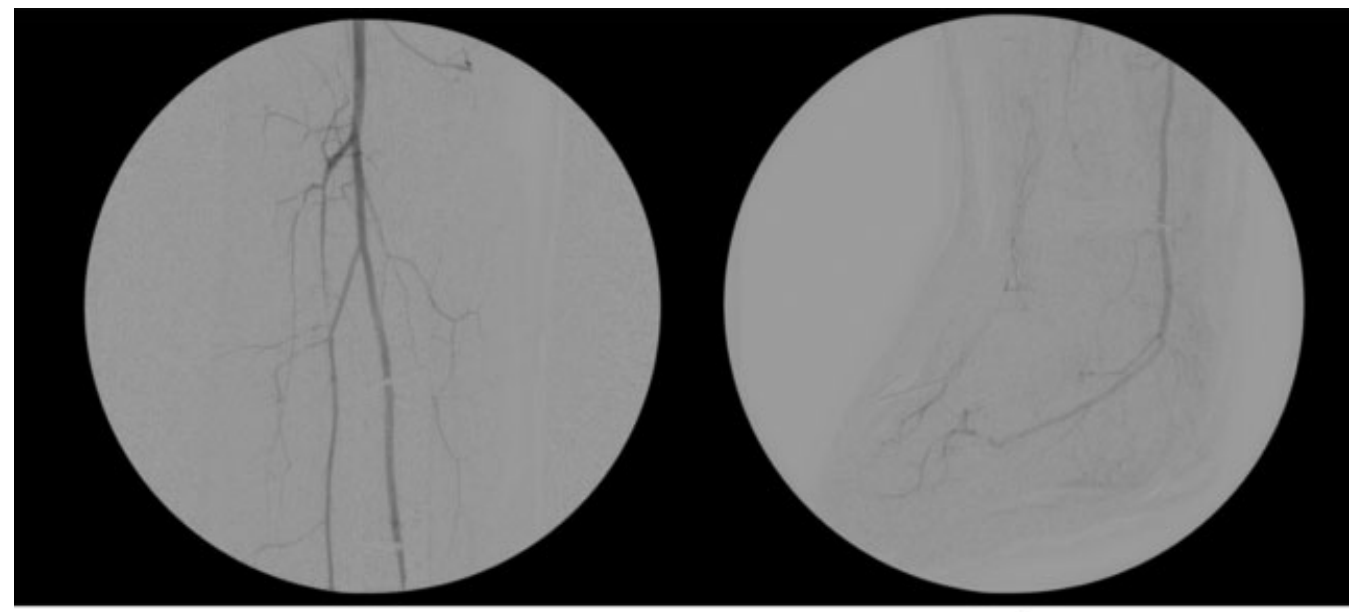

a

b

Fig. 2 Preoperative angiography: (a) preoperative anteroposterior view of leg, with early occlusion of the anterior tibial artery; and (b) lateral view of foot and ankle, with single vessel runoff to the foot via the posterior tibial artery.

fixed-rate heparin drip of 500 units per hour. He was transferred to the intensive care unit for hourly Doppler checks. He recovered uneventfully, and on hospital day 17 (POD 4) the heparin infusion was discontinued and he was transferred back to the general surgical floor in stable condition with a healthy viable flap and foot (-Fig. 4a,b). At the time of hospital discharge (postoperative day 8), both his flap and his foot remained healthy and viable. As of his most recent follow-up ( 5 months after surgery), his flap is viable and well-healed, and his right lower extremity has been successfully salvaged and remains neurovascularly intact, allowing for ambulation (-Fig. 5a,b).

\section{Discussion}

Despite need for conservation of resources and changing practice patterns amid the COVID-19 pandemic, there still exist indications for microsurgical free-flap reconstruction, including limb salvage. Recent literature describes a hypercoagulable state induced by COVID-19, with resultant catastrophic thrombotic events. While venous thrombotic events (DVT and PE) seem to be most common, there are also concerning reports of acute limb ischemia, cerebrovascular events in otherwise healthy patients, and aortic thromboses, of both natural arteries and previously patent bypass grafts. $^{2-9}$ It has also been shown that blood coagulation markers of COVID-19-positive patients differ significantly from healthy controls, indicating derangement of the coagulation cascade. ${ }^{10,11}$ These concerning reports must be considered when planning microsurgical reconstruction. Anastomoses may be at higher risk of both venous and arterial occlusion, increasing risk for flap loss. There is currently no data on microsurgical reconstruction in COVID-19-positive patients except for a single case report

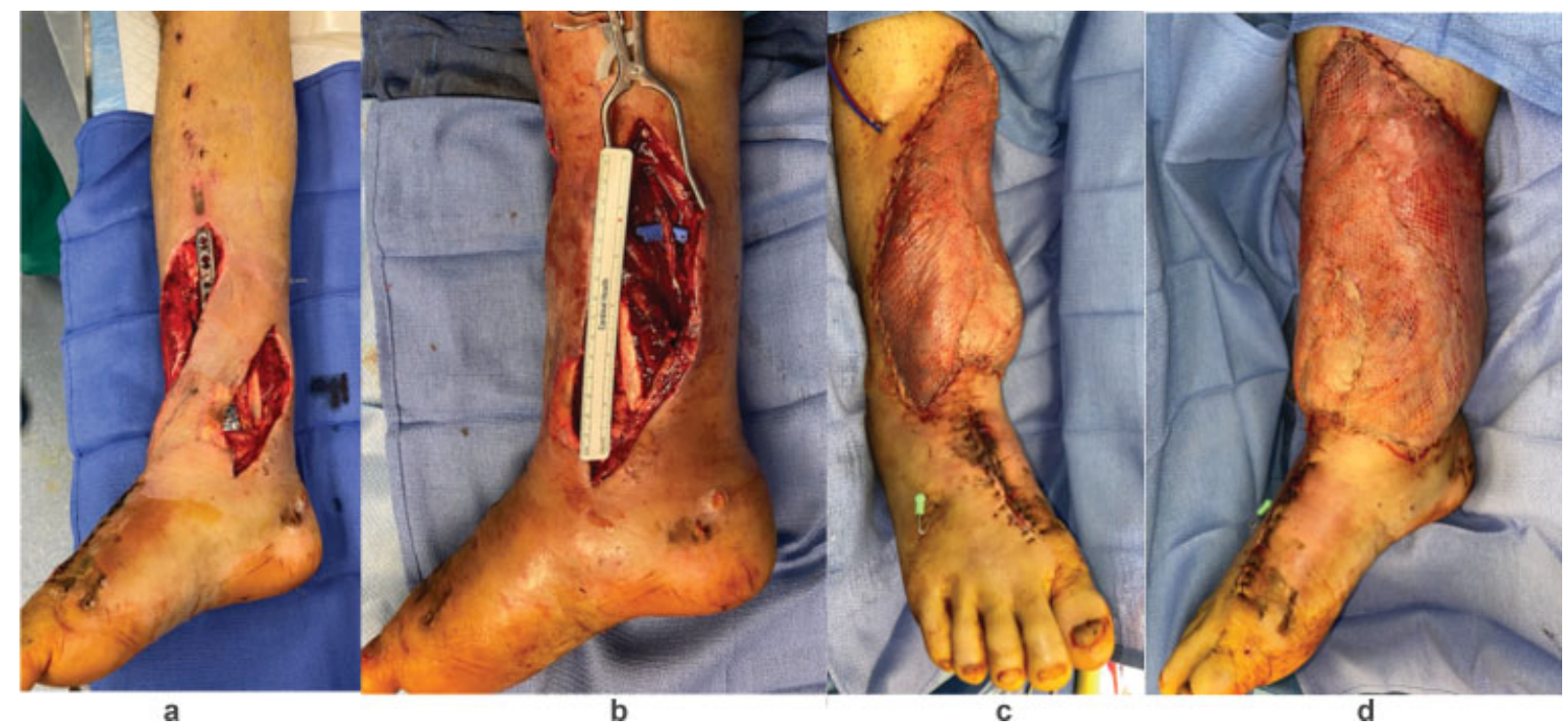

Fig. 3 Intraoperative free-flap photos: (a) preoperative defect with exposed orthopaedic hardware; (b) exposure of posterior tibial artery and veins; and (c, d) flap after inset and skin grafting. 


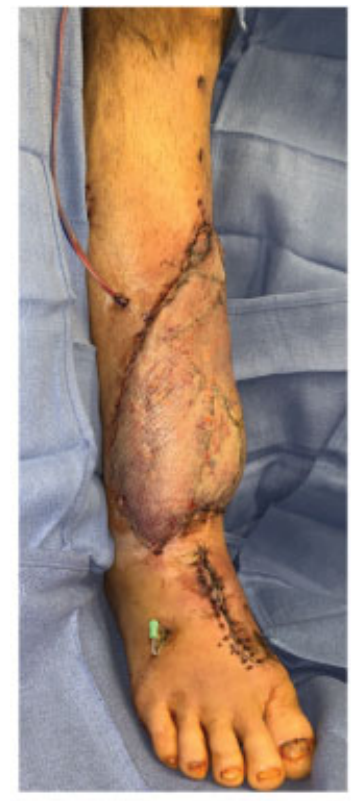

a

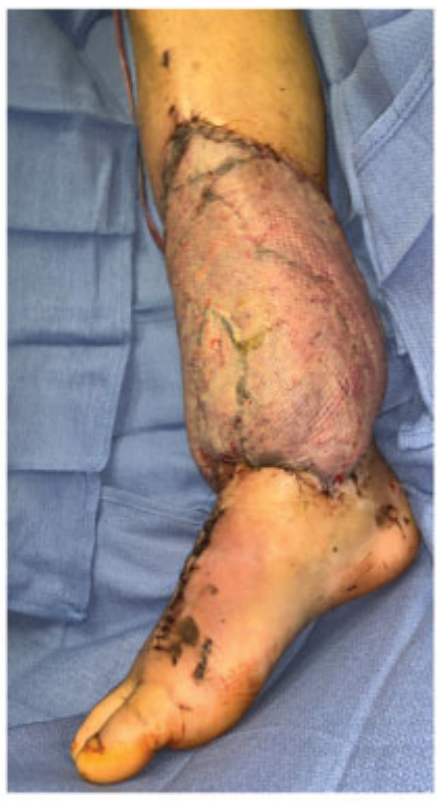

b
Fig. 4 (a, b) Postoperative day 4, healthy free flap with strong venous and arterial signals throughout.

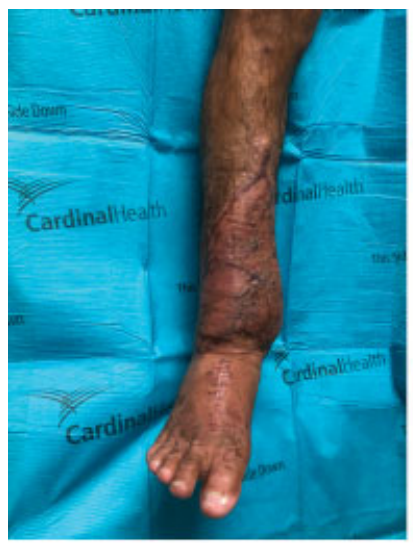

a

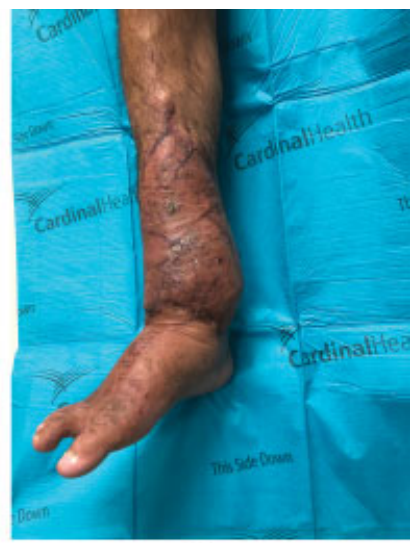

b
Fig. 5 Appearance of right lower extremity free flap 5 months after surgery. (a) Anteroposterior view. (b) Lateral oblique view.

of an unsuccessful flap for head and neck reconstruction, and a single report of a successful free flap in a patient found postoperatively to be COVID-19-positive. ${ }^{12,14}$ To our knowledge, ours was the first successful case of microsurgical freetissue transfer performed in a known COVID-19-positive patient to be reported in the literature.

It should be noted that at the time of our intervention, our nation's general experience with COVID-19 was extremely limited, as we were relatively early in the pandemic. It was not until after our intervention that data were published in Lancet indicating increased morbidity and mortality of surgery and intubation in patients with COVID-19. ${ }^{13}$ Had the data been available, they may well have altered our treatment course in favor of attempting to temporize coverage in the form of negative pressure wound therapy. However, this would have required frequent changes, risking contamination of the hardware, and subsequent infection and limb loss.
Instead, the risks and benefits were heavily weighed, and given the limited knowledge at the time, extensive discussions were performed between our team, our anesthesiologists and critical care physicians, and the patient, and the joint decision was made to proceed with surgery in an effort to salvage his limb. There continue to be difficult decisions regarding how and when to triage plastic surgery cases and patients in the time of COVID-19; to this end, Chi et al recently proposed a set of useful tier-based triage guidelines to assist with these difficult decisions. ${ }^{15}$ The highest tier in their proposed system (Tiers $3 \mathrm{~A}$ and $3 \mathrm{~B}$ ) encompasses healthy or unhealthy patients with high acuity problems, including open wounds at risk for infection, and wounds with exposed critical structures. The authors advocate for treating these patients without delay. Our patient's large leg wound with exposed orthopaedic hardware would certainly fall into the highest tier of this classification system, requiring urgent coverage.

Given the size and location of the defect, the latissimus dorsi muscle flap was chosen. Given our patient's contralateral foot injuries, we felt that limb salvage was critical and that expeditious coverage would minimize risk of hardware infection and subsequent nonunion and possible limb loss. With an absent anterior tibial artery (likely secondary to injury) and single-vessel runoff into his foot, the decision was made to perform end-to-side anastomosis into the posterior tibial artery. Two venous couplers were used for outflow anastomoses. We considered anastomosing end-to-end into the peroneal artery, but the obligatory fibulectomy would have required fibular nail removal, with subsequent destabilization of his syndesmotic injury.

Our postoperative protocol consisted of bedrest for 4 days, hourly Doppler checks by nursing, daily aspirin $325 \mathrm{mg}$ orally for a total of 30 days, and a fixed-rate heparin drip at 500 units per hour continued for 4 days postoperatively. This differs from our typical postoperative regimen for lower extremity free flaps in the trauma population, which consists only of aspirin $325 \mathrm{mg}$ daily orally for 30 days and prophylactic subcutaneous heparin. It should again be noted that at the time of this case, little was known or published about the optimal treatment of the hypercoagulable state associated with active COVID-19 infection. We did obtain a hematology consultation, but they were unable to provide any formal anticoagulation recommendations given the lack of available evidence, and instead deferred to the surgical team. Therefore, we extrapolated a management protocol from our department's experience performing free flaps on a large volume of patients with chronic lower extremity wounds, many of whom are thrombophilic. DeFazio et al studied the role intravenous heparin in these hypercoagulable patients and found that intravenous heparin infusion after surgery decreases thrombotic complications postoperatively in this thrombophilic population. ${ }^{16}$ In that study, hypercoagulable patients receiving lower extremity free flaps were treated with fixed-rate intravenous heparin infusions at 500 units per hour, and were increased to full therapeutic partial thromboplastin time (PTT)-driven anticoagulation only if there was 
significant calcification of the recipient vessels, or intraoperative anastomotic thromboses or revisions. Given this, we decided on a fixed-rate heparin dose postoperatively for our patient, and fortunately did not experience a thrombotic complication. However, this is clearly an area that would benefit from further study.

\section{Conclusion}

Although recent literature suggests increased incidence of venous and arterial thrombotic events in COVID-19-positive patients, microsurgical reconstruction is occasionally the lone option for salvage. We present a case of successful microsurgical free-flap reconstruction in a COVID-19-positive patient with right leg open Gustilo IIIb tibial and fibular fractures. Microsurgical reconstruction in this population must be carefully considered, meticulously executed, and closely monitored.

\section{Financial Disclosure Statement}

The authors have no financial disclosures.

Conflict of Interest

None declared.

\section{References}

1 Schoenbrunner AR, Sarac BA, Janis JE. A summary of recommendations for plastic surgeons during the Coronavirus disease 2019 outbreak. Plast Reconstr Surg Glob Open 2020;8(07):e3039

2 Kashi M, Jacquin A, Dakhil B, et al. Severe arterial thrombosis associated with Covid-19 infection. Thromb Res 2020;192:75-77

3 Klok FA, Kruip MJHA, van der Meer NJM, et al. Incidence of thrombotic complications in critically ill ICU patients with COVID-19. Thromb Res 2020;191:145-147
4 Bellosta R, Luzzani L, Natalini G, et al. Acute limb ischemia in patients with COVID-19 pneumonia. J Vasc Surg 2020;72(06): 1864-1872

5 Giacomelli E, Dorigo W, Fargion A, Calugi G, Cianchi G, Pratesi C. Acute thrombosis of an aortic prosthetic graft in a patient with severe COVID-19-related pneumonia. Ann Vasc Surg 2020;66:8-10

6 Gomez-Arbelaez D, Ibarra-Sanchez G, Garcia-Gutierrez A, Comanges-Yeboles A, Ansuategui-Vicente M, Gonzalez-Fajardo JA. COVID-19-related aortic thrombosis: a report of four cases. Ann Vasc Surg 2020;67:10-13

7 Vacirca A, Faggioli G, Pini R, Teutonico P, Pilato A, Gargiulo M. Unheralded lower limb threatening ischemia in a COVID-19 patient. Int J Infect Dis 2020;96:590-592

8 Ranasinghe V, Mady LJ, Kim S, et al. Major head and neck reconstruction during the COVID-19 pandemic: the University of Pittsburgh approach. Head Neck 2020;42(06):1243-1247

9 Mestres G, Puigmacià R, Blanco C, Yugueros X, Esturrica M, Riambau V. Risk of peripheral arterial thrombosis in COVID-19. J Vasc Surg 2020;72(02):756-757

10 Han H, Yang L, Liu R, et al. Prominent changes in blood coagulation of patients with SARS-CoV-2 infection. Clin Chem Lab Med 2020; 58(07):1116-1120

11 Zhang Y, Xiao M, Zhang S, et al. Coagulopathy and antiphospholipid antibodies in patients with Covid-19. N Engl J Med 2020;382 (17):e38

12 Benmoussa N, de Kerangal Q, Leymarie N, et al. Failure of free flaps in head and neck oncology surgery in COVID-19 patients. Plast Reconstr Surg 2020;146(02):251e-252e

13 Vuthaluru S, Koliyadan SV, Kanniwadi SS. Surgery during the COVID-19 pandemic. Lancet 2020;396(10261):e78

14 Lhuaire M, Garrido I, Cladière-Nassif V, Dumaine V, Lantieri L. Reconstructive microsurgery emergency in a patient with COVID-19. Plast Reconstr Surg 2020;146(04):523e-525e

15 Chi D, Chen AD, Dorante MI, Lee BT, Sacks JM. Plastic surgery in the time of COVID-19. J Reconstr Microsurg 2021;37(02):124-131

16 DeFazio MV, Economides JM, Anghel EL, Tefera EA, Evans KK. Lower extremity free tissue transfer in the setting of thrombophilia: analysis of perioperative anticoagulation protocols and predictors of flap failure. J Reconstr Microsurg 2019;35(04):270-286 